\title{
An ILP Formulation for Minimizing the Number of Feedthrough Cells in a Standard Cell Placement
}

\author{
JIN-TAI YAN \\ Computer Systems Research Center, National Chiao Tung University, Hsinchu, Taiwan, R.O.C.
}

(Received 17 November 1996; In final form 20 March 1998)

\begin{abstract}
It is well known that standard cells have been widely used to implement VLSI circuits in the automation of physical design. Since one major aim of a cell-based design is to minimize total layout area in a standard cell placement, the number of feedthrough cells will be minimized to reduce total cell area in a standard cell placement. In this paper, first, we model a partitioning-based row assignment (PRA) problem to minimize the number of feedthrough cells in a standard cell placement. Furthermore, an integer linear programming (ILP) approach is proposed to solve the PRA problem in a standard cell placement. Finally, the ILP approach has been implemented and two standard-cell netlists, Primary 1 and Primary 2, have been tested by the proposed approach, Bose's approach [4] and an exhaustive search approach, respectively. The experimental results show that the ILP approach obtains fewer feedthrough cells than Bose's approach in a partitioning-based standard cell placement.
\end{abstract}

Keywords: Physical design, placement, standard cell, feedthrough cell, integer linear programming

\section{INTRODUCTION}

It is well known that standard cells have been widely used to implement VLSI circuits in the automation of physical design. Due to inherent complexity of physical design in a standard cell design, this automation is further divided into two phases: placement and routing. In the placement phase, all the standard cells in a netlist are arranged within several horizontal rows with minimum wire length $[1-6]$. Furthermore, in the routing phase, a global routing process adds feedthrough cells [6-9] into cell rows to make all the global nets to be connected, and a detailed routing process completes all the local connections in any channel by a detailed router. Although physical design in a cell-based design is divided into two continuous phases, these two phases are seriously relative. For a cell-based design, the routing configuration always depends on the placement result. For example, the number of feedthrough cells in a standard cell placement is decided by the positions of the cells in the placement result. In Figure 1, a graphical representation of a cell netlist, a standard cell placement, and the insertion of a feedthrough cell in this placement are shown. 


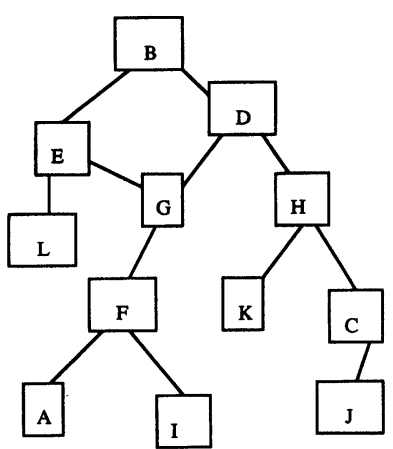

(a)

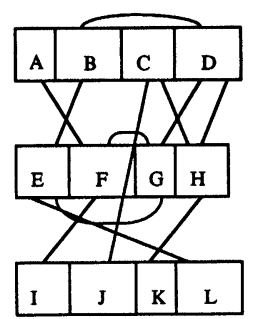

(b)

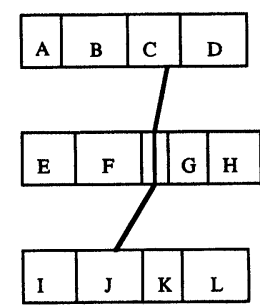

(c)

FIGURE 1 A cell netlist and its standard cell placement. (a) A graphical representation of a cell netlist. (b) A standard cell placement. (c) The insertion of a feedthrough cell in a standard cell placement.

Since one major aim of a cell-based design is to minimize total layout area in a standard cell placement, it is important for the minimization of total layout area to minimize total cell area in a standard cell placement. Basically, the cells in a standard cell placement are divided into standard cells and feedthrough cells. To our knowledge, standard cells are used to implement VLSI circuits in a circuit netlist. Hence, it is impossible that the number of standard cells in a cell netlist is minimized in the automation of physical design. In contrast, feedthrough cells are used to connect all the global nets in a standard cell placement. The number of feedthrough cells can be minimized to reduce total cell area by an optimal cell assignment in a standard cell placement. It is no wonder that a standard cell placement is in a linear multi-row structure. The connection of all the global nets in a standard cell placement is defined by the decision of a row ordering. Therefore, it plays an important role for the minimization of the number of feedthrough cells [3-4] to obtain an optimal row assignment in a standard cell placement.

Traditionally, a standard cell placement is obtained by two different approaches: a linearassignment-based approach [10-14] and a partitioning-based approach [1-6]. In a linearassignment-based approach, standard cells in a cell netlist are first linearized to minimize the wire length or the number of crossings by a linearizing operation. Schuler and Ulrich [10] first proposed the concept of clustering and a linear placement for a cell-based placement, and a linear-placement problem was transformed into a linear-embedding problem. Unfortunately, the linear-embedding problem [11] was proved to be NP-complete. Later, for the linear-placement problem, Chowdhury [12] proposed an analytical approach to obtain an approximate solution, and some heuristic approaches [13-14] were proposed to obtain several near-optimal solutions.

For a two-dimensional placement, according to an aspect ratio and a row number $K$ in a desired placement, Kang [14] first applied the idea of folding a linear placement to form $K$ cell rows in a standard cell placement. Consider the net connection in a cell netlist, a standard cell placement folded from a one-dimensional placement may not be a good two-dimensional row-based placement, and the introduction of feedthrough cells in a standard cell placement is not explicitly addressed by a linear-assignment-based approach. Clearly, the minimization of the number of feedthrough cells in a standard cell placement is not considered in the row assignment step for a linear-assignmentbased approach.

Recently, a partitioning-based approach [1$5,16,17]$ has been widely applied to obtain a standard cell placement. First, according to an aspect ratio and a row number $K$ in a desired placement, all the standard cells in a cell netlist are partitioned into $K$ groups by a hierarchical or multi-way partitioning operation. For a partitioning-based standard cell placement, a $K$ and 
$L$-based two-way min-cut partitioning by Dunlop and Kernighan [1], a quadrisection partitioning by Suaris and Kedem [2], and a two-way minimum ratio-cut partitioning by Hamada, Cheng and Chau [5] were proposed to hierarchically partition a cell netlist into smaller cell netlists, respectively. However, these partitioning-based approaches focus on the performance of the partitioning result, and the minimization of the number of feedthrough cells in a standard cell placement is not considered in the placement of standard cells.

For the introduction of feedthrough cells in a partitioning-based approach, to our knowledge, the problem of minimizing the number of feedthrough cells in a standard cell placement has been proved to be a NP-complete problem [11]. Cho and Kyung [3] first proposed a heuristic algorithm based on a constrained multi-stage graph to minimize the number of feedthrough cells in a standard cell placement. Later, Bose and Saha [4] also proposed a combined partitioning-based row assignment to minimize the number of feedthrough cells in a standard cell placement. However, these two algorithms [3-4] first partition a cell netlist into two smaller netlists by a $K$ and $L$-based partitioning, and the further netlist partitioning is involved in the row assignment step. Clearly, the number of feedthrough cells in a standard cell placement depends on the selection of seed nets in a netlist partitioning. Hence, the minimization of the number of feedthrough cells in a partitioningbased row assignment is decided by a hierarchical performance of $K$ and $L$-based partitioning.

According to the definition of a partitioningbased approach, we model a new partitioningbased approach for a standard cell placement. Basically, this approach is divided into three phases: multi-way netlist partitioning, row assignment (inter-row placement) and multi-row linear placement (intra-row placement). In multi-way netlist partitioning, according to a row number $K$ in a desired placement and an area bound in any row, standard cells in a cell netlist are partitioned into $K$ smaller netlists by a multi-way area-ratioconstrained (ARC) partitioning [15]. In a row assignment, $K$ netlists are vertically linearized to minimize the number of feedthrough cells for a standard cell placement. Finally, $K$ netlists are horizontally linearized to be $K$ cell rows to minimize total channel density in a multi-row linear placement.

In this paper, first, we model a partitioningbased row assignment (PRA) problem to minimize the number of feedthrough cells in a standard cell placement. Furthermore, an integer linear programming (ILP) approach is proposed to solve the PRA problem in a standard cell placement. Finally, the ILP approach has been implemented and two standard-cell netlists, Primary 1 and Primary 2, have been tested by the proposed approach, Bose's approach [4] and an exhaustive search approach, respectively. The experimental results show that the ILP approach obtains fewer feedthrough cells than Bose's approach in a partitioning-based standard cell placement.

\section{PROBLEM DESCRIPTION AND DEFINITIONS}

Before we model a partitioning-based row assignment (PRA) problem in a standard cell placement, some important assumptions will be described as follows: First, assume that each feedthrough cell only contains one vertical net segment crossing one cell row in a standard cell placement. Hence, the number of vertical net segments crossing any cell row in a standard cell placement is the number of feedthrough cells in the cell row. Second, if all the pins of a net are fully distributed onto two adjacent cell rows, the net is defined as a local net. In contrast, if all the pins of a net are not fully distributed onto two adjacent cell rows, the net is defined as a global net. Hence, it is assumed that the connection of a local net is free of any feedthrough cell in a standard cell placement. In contrast, the connection of a global net is obtained by adding feedthrough cells in a standard cell placement. Although the pins of a global net are 
distributed into different cell rows, it is assumed that each global net generates at most one feedthrough cell in each cell row in a standard cell placement. For a global net in a standard cell placement, the net will be separated into several local nets by assigning at most one feedthrough cell in each cell row in a standard cell placement. Refer to Figure 1(c), the connection of a global net in a standard cell placement is obtained by the assignment of a feedthrough cell.

Given a row number $K$ in a desired placement, based on the proposed partitioning-based approach, all the standard cells in a cell netlist are partitioned into $K$ groups by using a multi-way area-ratio-constrained partitioning. Furthermore, the number of feedthrough cells will be minimized to reduce total cell area by using a partitioningbased row assignment in a standard cell placement. Basically, $K$ cell groups in a multi-way area-ratio-constrained partitioning construct a separation graph for the PRA problem in a standard cell placement as follows:

Definition 1 For $K$ cell groups in a $K$-way arearatio-constrained partitioning, a separation graph $G(V, E)$ is an undirected edge-weighted graph defined as follows: any cell group in this $K$-way partitioning is represented as one vertex in $V$. Each edge $(u, v)$ is in $E$ if and only if two cell groups corresponding to $u$ and $v$ are connected by at least one net connection, and the weight $w(u, v)$ between $u$ and $v$ is the number of net connections between $u$ and $v$.

For a 4-way area-ratio-constrained partitioning in a cell netlist, 4 cell groups and a mapped separation graph are shown in Figure 2, where $V$ is $\left\{v_{1}, v_{2}, v_{3}, v_{4}\right\}, E$ is $\left\{\left(v_{1}, v_{2}\right),\left(v_{1}, v_{3}\right),\left(v_{1}, v_{4}\right),\left(v_{2}, v_{3}\right)\right.$, $\left.\left(v_{2}, v_{4}\right),\left(v_{3}, v_{4}\right)\right\}$ and $w\left(e_{1,2}\right)=1, w\left(e_{1,3}\right)=2, w\left(e_{1,4}\right)$ $=4, w\left(e_{2,3}\right)=3, w\left(e_{2,4}\right)=1$, and $w\left(e_{3,4}\right)=5$.

Due to a vertical linear structure in a standard cell placement, the PRA problem in a standard cell placement can be transformed into the problem of finding a linear embedding of a separation graph with minimum crossings. Hence, for a given graph, the crossing distance of any edge, and total

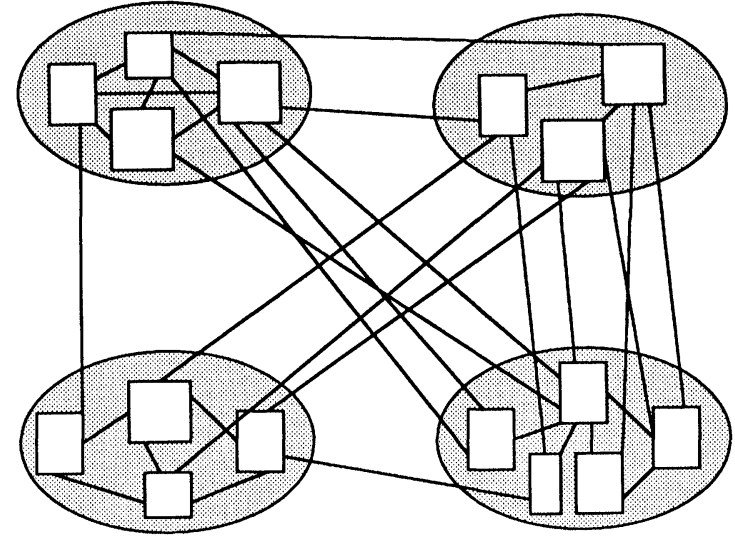

(a)

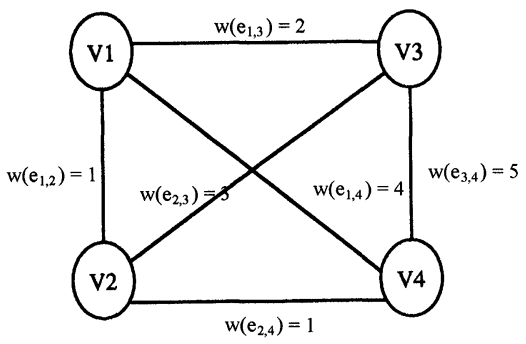

(b)

FIGURE 2 Area-ratio-constrained partitioning and separation graph. (a) 4-way area-ratio-constrained partitioning in a cell netlist. (b) A mapped separation graph of Figure 2(a).

crossing distance in a linear embedding will be defined as follows:

Definition 2 For an undirected graph $G(V, E)$, a linear embedding $E(G)$ of $G$ is defined as a linear ordering $\left(v_{1}, v_{2}, \ldots, v_{n-1}, v_{n}\right)$ of $V$. If $\left(v_{i}, v_{i+1}\right)$, $1 \leq i \leq n-1$, is in $E$, the edge $\left(v_{i}, v_{i+1}\right)$ is called as an embedding edge. In contrast, if $\left(v_{i}, v_{j}\right)$, $1 \leq i, j \leq n, i \neq j$, is in $E$ and not an embedding edge, the edge $\left(v_{i}, v_{j}\right)$ is called as a non-embedding edge.

Definition 3 For a linear embedding $E(G)$ of $G$, the crossing distance $\operatorname{Dist}(u, v)$ of any edge $(u, v)$ in $G$ will be defined as

$$
\operatorname{Dist}(u, v)=w(u, v) * \operatorname{Dif}(u, v)
$$


where $\operatorname{Dif}(u, v)$ is the number of vertices between $u$ and $v$ in $E(G)$. Furthermore, total crossing distance in $E(G)$ will be defined as the sum of the crossing distances of all the edges in $G$ such that

$$
\text { Total_Dist }=\sum_{(u, v) \text {.in. } E} \operatorname{Dist}(u, v) .
$$

According to the previous assumptions and definitions, the PRA problem of minimizing the number of feedthrough cells in a standard cell placement corresponds to the linear embedding (LE) problem of minimizing total crossing distance for a separation graph. Unfortunately, the LE problem of minimizing total crossing distance for a separation graph is still a NP-complete problem [18]. Hence, no optimal polynomial approach is proposed to solve the LE problem.

\section{ILP FORMULATION FOR MINIMIZATION OF THE NUMBER OF FEEDTHROUGH CELLS IN A STANDARD CELL PLACEMENT}

As mentioned above, the PRA problem of minimizing the number of feedthrough cells in a standard cell placement corresponds to the linear embedding (LE) problem of minimizing total crossing distance for a separation graph. We propose a new integer linear programming (ILP) formulation to guarantee an optimal solution for the PRA problem in a standard cell placement as follows:

If a separation graph $G(V, E)$ is not a complete graph, this graph will be firstly modified into a complete separation graph by adding zeroweighted edges. Hence, according to the computation of the edge weights of $E=\left\{e_{i, j} \mid 1 \leq i, j \leq n\right.$, $i<j\}$ in $G$, the LE problem of minimizing total crossing distance for a separation graph is to find an permutation of $V=\left\{v_{1}, v_{2}, \ldots, v_{n}\right\}$ on $n$ consecutive locations $\{1,2, \ldots, n\}$ with minimum total crossing distance.
In ILP formulation, two groups of variables $x_{i, j}$ and $y_{i, j, k}$ are introduced to model a linear objective function for total crossing distance in the LE problem, where

$x_{i, j}=1, \quad$ if $v_{i}$ is placed in location $j$, otherwise $x_{i, j}=0$.

$y_{i, j, k}=1, \quad$ if the difference of the locations of $v_{i}$ and $v_{j}$ is $k$,otherwise $y_{i, j, k}=0$.

Hence, the objective function to be minimized for total crossing distance in the LE problem is

$$
\sum_{i=1}^{n} \sum_{j>i}^{n} \sum_{k=2}^{n-1}(k-1) w\left(e_{i j}\right) y_{i, j, k}
$$

subject to

$$
\sum_{j=1}^{n} x_{i, j}=1, \quad \text { for } 1 \leq i \leq n
$$

$$
\sum_{i=1}^{n} x_{i, j}=1, \quad \text { for } 1 \leq j \leq n
$$

$y_{i, j, k}=y_{j, i, k}, \quad$ for $1 \leq i, j \leq n, i<j, 0<k<n$,

$$
\begin{aligned}
& \sum_{k=1}^{n-1} y_{i, j, k}=1, \quad \text { for } 1 \leq i, j \leq n, \quad i<j, \\
& \sum_{j=1, j \neq i}^{n} y_{i, j, k} \leq 2, \quad \text { for } 1 \leq i \leq n, 0<k<n / 2
\end{aligned}
$$

$$
\sum_{j=1, j \neq i}^{n} y_{i, j, k} \leq 1, \quad \text { for } 1 \leq i \leq n, n / 2 \leq k<n
$$

$$
\begin{aligned}
& \sum_{k=1}^{n-1} k y_{i, j, k}-\sum_{k=1}^{n} k x_{i, k}+\sum_{k=1}^{n} k x_{j, k} \geq 0 \\
& \quad \text { for } 1 \leq i, j \leq n, i<j .
\end{aligned}
$$


For the constraints of this objective function, constraint (2) states that each vertex must be placed on one of $n$ locations. In contrast, constraint (3) states that each location only places one of $n$ vertices. The two constraints show a one-toone mapping relation between vertices and locations. Clearly, for $n$ vertices, there are $n$ ! conditions in such a one-to-one mapping function. Constraint (4) denotes a symmetric property that the distance of the locations of $v_{i}$ and $v_{j}$ is $k$. It is clear that the distance from the location of $v_{i}$ to the location of $v_{j}$ is equal to that from the location of $v_{j}$ to the location of $v_{i}$. Constraint (5) states that the distance of the locations of $v_{i}$ and $v_{j}$ is at least 1 and at most $n-1$. Since $v_{i}$ and $v_{j}$ are located on two different fixed locations, the distance of the locations of $v_{i}$ and $v_{j}$ is unique. Constraints (6) $-(7)$ denote a linear embedding relation between $v_{i}$ and $v_{j}$ with a fixed distance $k$. For a distance $k$, $0<k<n / 2$, the number of the vertices the distance of which and $v_{i}$ is $k$ is at most 2 . In contrast, for a distance $k, n / 2 \leq k<n$, the number of the vertices the distance of which and $v_{i}$ is $k$ is at most 1. Finally, according to the distance of the locations of $v_{i}$ and $v_{j}$, constraint (8) denotes a distance relation between two groups of variables $x_{i, j}$ and $y_{i, j, k}$.

Refer to Figure 2, an ILP formulation is modeled to solve the LE problem of minimizing total crossing distance for a separation graph as follows:

$$
\text { Minimize } \quad \begin{aligned}
y_{1,2,2} & +2 y_{1,3,2}+4 y_{1,4,2}+3 y_{2,3,2}+y_{2,4,2} \\
& +5 y_{3,4,2}+2 y_{1,2,3}+4 y_{1,3,3}+8 y_{1,4,3} \\
& +6 y_{2,3,3}+2 y_{2,4,3}+10 y_{3,4,3}
\end{aligned}
$$

subject to

$$
\begin{aligned}
& x_{1,1}+x_{1,2}+x_{1,3}+x_{1,4}=1, x_{2,1}+x_{2,2}+x_{2,3}+x_{2,4}=1, \\
& x_{3,1}+x_{3,2}+x_{3,3}+x_{3,4}=1, x_{4,1}+x_{4,2}+x_{4,3}+x_{4,4}=1, \\
& x_{1,1}+x_{2,1}+x_{3,1}+x_{4,1}=1, x_{1,2}+x_{2,2}+x_{3,2}+x_{4,2}=1, \\
& x_{1,3}+x_{2,3}+x_{3,3}+x_{4,3}=1, x_{1,4}+x_{2,4}+x_{3,4}+x_{4,4}=1,
\end{aligned}
$$

$$
\begin{aligned}
& y_{1,2,1}=y_{2,1,1}, \quad y_{1,2,2}=y_{2,1,2}, \quad y_{1,2,3}=y_{2,1,3}, \\
& y_{1,3,1}=y_{3,1,1}, \quad y_{1,3,2}=y_{3,1,2}, \quad y_{1,3,3}=y_{3,1,3}, \\
& y_{1,4,1}=y_{4,1,1}, \quad y_{1,4,2}=y_{4,1,2}, \quad y_{1,4,3}=y_{4,1,3}, \\
& y_{2,3,1}=y_{3,2,1}, \quad y_{2,3,2}=y_{3,2,2}, \quad y_{2,3,3}=y_{3,2,3}, \\
& y_{2,4,1}=y_{4,2,1}, \quad y_{2,4,2}=y_{4,2,2,}, y_{2,4,3}=y_{4,2,3}, \\
& y_{3,4,1}=y_{4,3,1}, \quad y_{3,4,2}=y_{4,3,2}, \quad y_{3,4,3}=y_{4,3,3}, \\
& y_{1,2,1}+y_{1,2,2}+y_{1,2,3}=1, \quad y_{1,3,1}+y_{1,3,2}+y_{1,3,3}=1, \\
& y_{1,4,1}+y_{1,4,2}+y_{1,4,3}=1, \quad y_{2,3,1}+y_{2,3,2}+y_{2,3,3}=1, \\
& y_{2,4,1}+y_{2,4,2}+y_{2,4,3}=1, \quad y_{3,4,1}+y_{3,4,2}+y_{3,4,3}=1, \\
& y_{1,2,1}+y_{1,3,1}+y_{1,4,1} \leq 2, \quad y_{1,2,2}+y_{1,3,2}+y_{1,4,2} \leq 1, \\
& y_{1,2,3}+y_{1,3,3}+y_{1,4,3} \leq 1, \quad y_{2,1,1}+y_{2,3,1}+y_{2,4,1} \leq 2, \\
& y_{2,1,2}+y_{2,3,2}+y_{2,4,2} \leq 1, \quad y_{2,1,3}+y_{2,3,3}+y_{2,4,3} \leq 1, \\
& y_{3,1,1}+y_{3,2,1}+y_{3,4,1} \leq 2, \quad y_{3,1,2}+y_{3,2,2}+y_{3,4,2} \leq 1, \\
& y_{3,1,3}+y_{3,2,3}+y_{3,4,3} \leq 1, \quad y_{4,1,1}+y_{4,2,1}+y_{4,3,1} \leq 2, \\
& y_{4,1,2}+y_{4,2,2}+y_{4,3,2} \leq 1, \quad y_{4,1,3}+y_{4,2,3}+y_{4,3,3} \leq 1, \\
& y_{1,2,1}+2 y_{1,2,2}+3 y_{1,2,3}-\left(x_{1,1}+2 x_{1,2}+3 x_{1,3}+4 x_{1,4}\right) \\
& +\left(x_{2,1}+2 x_{2,2}+3 x_{2,3}+4 x_{2,4}\right) \geq 0, \\
& y_{1,3,1}+2 y_{1,3,2}+3 y_{1,3,3}-\left(x_{1,1}+2 x_{1,2}+3 x_{1,3}+4 x_{1,4}\right) \\
& +\left(x_{3,1}+2 x_{3,2}+3 x_{3,3}+4 x_{3,4}\right) \geq 0, \\
& y_{1,4,1}+2 y_{1,4,2}+3 y_{1,4,3}-\left(x_{1,1}+2 x_{1,2}+3 x_{1,3}+4 x_{1,4}\right) \\
& +\left(x_{4,1}+2 x_{4,2}+3 x_{4,3}+4 x_{4,4}\right) \geq 0, \\
& y_{2,3,1}+2 y_{2,3,2}+3 y_{2,3,3}-\left(x_{2,1}+2 x_{2,2}+3 x_{2,3}+4 x_{2,4}\right) \\
& +\left(x_{3,1}+2 x_{3,2}+3 x_{3,3}+4 x_{3,4}\right) \geq 0, \\
& +2 y_{2,4,2}+3 y_{2,4,3}-\left(x_{2,1}+2 x_{2,2}+3 x_{2,3}+4 x_{2,4}\right) \\
& +\left(x_{4,1}+2 x_{4,2}+3 x_{4,3}+4 x_{4,4}\right) \geq 0, \\
& +\left(x_{4,1,2}+2 x_{4,2}+3 x_{4,3}+4 x_{4,4}\right) \geq 0 .
\end{aligned}
$$

For a separation graph in Figure 2, this ILP formulation obtains a linear embedding $\left(v_{1}, v_{4}, v_{3}\right.$, $v_{2}$ ) of $\left\{v_{1}, v_{2}, v_{3}, v_{4}\right\}$, and the embedding is optimal. From a vertex ordering in $\left(v_{1}, v_{4}, v_{3}, v_{2}\right)$, the minimum total crossing distance in $\left(v_{1}, v_{4}, v_{3}, v_{2}\right)$ is 5 , that is, the number of feedthrough cells in a standard cell placement is 5 .

\section{EXPERIMENTAL RESULTS}

For the PRA problem of minimizing the number of feedthrough cells in a standard cell placement, 
TABLE I Best cut and average cut of primary 1 and primary 2 in 20 runs for $K=4,8,12,16$ and 20

\begin{tabular}{lcccccccccr}
\hline & \multicolumn{2}{c}{$K=4$} & \multicolumn{2}{c}{$K=8$} & \multicolumn{2}{c}{$K=12$} & \multicolumn{2}{c}{$K=16$} & \multicolumn{2}{c}{$K=20$} \\
\cline { 2 - 11 } Example & $\begin{array}{c}\text { Best } \\
\text { mincut }\end{array}$ & $\begin{array}{c}\text { Avg. } \\
\text { mincut }\end{array}$ & $\begin{array}{c}\text { Best } \\
\text { mincut }\end{array}$ & $\begin{array}{c}\text { Avg. } \\
\text { mincut }\end{array}$ & $\begin{array}{c}\text { Best } \\
\text { mincut }\end{array}$ & $\begin{array}{c}\text { Avg. } \\
\text { mincut }\end{array}$ & $\begin{array}{c}\text { Best } \\
\text { mincut }\end{array}$ & $\begin{array}{c}\text { Avg. } \\
\text { mincut }\end{array}$ & $\begin{array}{c}\text { Best } \\
\text { mincut }\end{array}$ & $\begin{array}{c}\text { Avg. } \\
\text { mincut }\end{array}$ \\
\hline Primary1 & 81 & 92.3 & 173 & 197.6 & 263 & 307.5 & 372 & 413.8 & 494 & 579 \\
Primary2 & 234 & 283.7 & 458 & 497.5 & 715 & 782.1 & 954 & 1084.6 & 1267 & 1394 \\
\hline
\end{tabular}

TABLE II Experimental results for primary 1 and primary 2

\begin{tabular}{|c|c|c|c|c|c|c|c|c|c|c|}
\hline \multirow[b]{3}{*}{ \#Rows } & \multicolumn{5}{|c|}{ Primary 1} & \multicolumn{5}{|c|}{ Primary 2} \\
\hline & \multicolumn{2}{|c|}{ Bose [4] } & \multicolumn{2}{|c|}{ ILP } & \multirow{2}{*}{$\begin{array}{c}\begin{array}{c}\text { Exhaustive } \\
\text { search }\end{array} \\
\text { \#Cell }\end{array}$} & \multicolumn{2}{|c|}{ Bose [4] } & \multicolumn{2}{|r|}{ ILP } & \multirow{2}{*}{$\begin{array}{c}\text { Exhaustive } \\
\text { search }\end{array}$} \\
\hline & \#Cell & Time(s) & \#Cell & Time(s) & & \#Cell & Time(s) & \#Cell & Time(s) & \\
\hline & 44 & 0.3 & 36 & 0.1 & 36 & 135 & 0.4 & 117 & 0.1 & 117 \\
\hline 8 & 126 & 0.9 & 108 & 0.5 & 108 & 364 & 1.4 & 324 & 0.5 & 324 \\
\hline 12 & 228 & 2.1 & 195 & 1.5 & 195 & 642 & 2.5 & 569 & 1.6 & 569 \\
\hline 16 & 331 & 5.9 & 286 & 4.3 & 286 & 872 & 6.1 & 785 & 4.5 & 785 \\
\hline 20 & 487 & 10.7 & 414 & 8.3 & 414 & 1271 & 11.2 & 1102 & 8.4 & 1102 \\
\hline
\end{tabular}

*Bose's time includes the time of netlist partitioning.

the ILP approach has been implemented using the LINDO [21] package. On the other hand, Bose's approach [4] and an exhaustive search approach have been implemented by using $C$ language on a SUN workstation under the Berkeley 4.2 UNIX operating system. Two standard-cell netlists, Primary 1 and Primary 2, have been tested by the ILP proposed approach, Bose's approach [4] and an exhaustive search approach, respectively.

Before doing partitioning-based row assignment in a standard cell placement, a standard cell netlist is partitioned into some clusters by a multi-way ARC partitioning. In a multi-way ARC min-cut partitioning [15], given a row number $K$, average partitioning area (APA) in one row is obtained as

$$
\mathrm{APA}=(\text { total cell area in a cell netlist }) / K .
$$

Furthermore, row partitioning area (RPA) in one cell row is constrained from $80 \%{ }^{*} \mathrm{APA}$ to $120 \%{ }^{*}$ APA, that is, $80 \%{ }^{*} \mathrm{APA} \leq \mathrm{RPA} \leq 120 \%{ }^{*}$ APA. For $K=4,8,12,16$, or 20 , two cell netlists, Primary 1 and Primary 2, are firstly partitioned into $4,8,12,16$, and 20 smaller netlists by a multi-way ARC min-cut partitioning [15], respectively. For Primary 1 and Primary 2, the best cut and the average cut in 20 runs are shown in Table I.
For Primary 1 and Primary 2, all the partitioning results with the best cut in $K=4,8,12,16$ and 20 are transformed into different separation graphs. Consider these Primary 1's and Primary 2's separation graphs, the ILP approach and an exhaustive search approach yield a linear embedding for any separation graph, respectively, and total crossing distance of each linear embedding is computed according to the definition of total crossing distance in a linear embedding. Hence, the number of feedthrough cells in a standard cell placement with $K$ rows is obtained by the computation of total crossing distance in a linear embedding. In Table II, the number of feedthrough cells and the running time in a standard cell placement with $4,8,12,16$ and 20 rows obtained by the proposed ILP approach, Bose's approach and an exhaustive search approach are shown. The experimental results show that the ILP approach obtains fewer feedthrough cells than Bose's approach in a partitioning-based standard cell placement.

\section{CONCLUSION}

In this paper, first, we model a partitioning-based row assignment (PRA) problem to minimize the 
number of feedthrough cells in a standard cell placement. Furthermore, an integer linear programming (ILP) approach is proposed to solve the PRA problem in a standard cell placement. Finally, the ILP approach has been implemented and two standard-cell netlists, Primary 1 and Primary 2, have been tested by the proposed approach, Bose's approach [4] and an exhaustive search approach, respectively. The experimental results show that the ILP approach obtains fewer feedthrough cells than Bose's approach in a partitioning-based standard cell placement.

\section{References}

[1] Dunlop, A. E. and Kernighan, B. W. (1985). "A Procedure for Placement of Standard Cell VLSI Circuits", IEEE Trans. on Computer-Aided Design, 4, 92-98.

[2] Suaris, P. R. and Kedem, G. (1988). "An Algorithm for Quadrisection and Its Application to Standard Cell Placement", IEEE Trans. on Circuit and Systems, 35, 294-303.

[3] Cho, H. G. and Kyung, C. M. (1988). "A Heuristic Standard Cell Placement Algorithm Using Constrained Multistage Graph Model", IEEE Trans. on ComputerAided Design, 7, 1205-1214.

[4] Bose, S. and Saha, A. R. (1993). "Implementation of A Heuristic Method for Standard Cell Placement", International Journal of Electronics, 74, 281-298.

[5] Hamada, T., Cheng, C. K. and Chau, P. M. (1992). "An Efficient MultiLevel Placement Technique Using Hierarchical Partitioning", IEEE Trans. on Circuit and Systems-I: Fundamental Theory and Applications, 39, $432-439$.

[6] Edahiro, M. and Yoshimura, T. (1990). "New Placement and Global Routing Algorithms for Standard Cell Layouts", Proc. 27th Design Automation Conference, pp. $642-645$.

[7] Cong, J. and Preas, B. (1992). "A New Algorithm for Standard Cell Global Routing", INTEGRATION, the VLSI journal, 14, 49-65.

[8] Hong, X. L., Huang, J., Cheng, C. K. and Kuh, E. S. (1992). "FARM: An Efficient Feed-Through Pin Assignment Algorithm", Proc. 29th Design Automation Conference, pp. $530-535$.

[9] Okamoto, T., Ishikawa, M. and Fujita, T. (1993). "A New Feed-Through Assignment Algorithm Based on a Flow Model", International Conference on Computer-Aided Design, pp. 775-778.

[10] Schuler, D. M. and Ulrich, E. G. (1972). "Clustering and Linear Placement", Proc. 9th Design Automation Conference, pp. 50-56.
[11] Garey, M. R. and Johnson, D. S. (1979). Computers and Intractability: A Guide to the Theory of NP-Completeness. San Francisco, CA: Freeman.

[12] Chowdhury, S. (1989). "Analytical Approaches to the Combinatorial Optimization in Linear Placement Problem", IEEE Trans. on Computer-Aided Design, 8, 630-639.

[13] Meixner, G. and Lauther, U. (1990). "A New Global Router Based on A Flow Model and Linear Assignment", International Conference on Computer-Aided Design.

[14] Kang, S. (1983). "Linear Ordering and Application to Placement", Proc. 20th Design Automation Conference, pp. 457-464.

[15] Yan, J. T. (1994). "Area-Ratio-Constrained Min-Cut Partitioning for Row-Based Placement", IEEE 37th Midwest Symposium on Circuits and Systems, MSCAS'94, pp. $403-406$.

[16] Mattison, R. L. (1974). "Design Automation of MOS Artwork", IEEE Trans. on Computers, 23, 21-27.

[17] Goto, S. and Kuh, E. S. (1978). "An Approach to the Two-Dimensional Placement Problem in Circuit Layout", IEEE Trans. on Circuits and Systems, 25.

[18] Garey, M. R. and Johnson, D. S. (1976). "Some Simplified NP-Complete Graph Problems", Theoretical Computer Science, 1, 237-267.

[19] Masuda, S., Nakajima, K., Kashiwabara, T. and Fujisawa, T. (1990). "Crossing Minimization in Linear Embeddings of Graphs", IEEE Trans. on Computers, 39, 123-127.

[20] Garfinkel, R. S. and Nemhauser, G. L. (1972). Integer Programming. New York: Wiley Interscience.

[21] "LINDO: Linear Interactive and Discrete Optimizer for Liner, Integer and Quadratic Programming Problems", LINDO System Inc.

\section{Author's Biography}

Jin-Tai Yan received the B.S., M.S., and Ph.D. degrees in computer and information science from National Chiao-Tung University, Hsinchu, Taiwan, R.O.C. in 1988, 1990, and 1995, respectively.

From 1995 to 1997 he served in the Chinese Navy, Kaohsiung, Taiwan, as an information officer. Since 1997 he has been with National Chiao-Tung University, Hsinchu, Taiwan, R.O.C. and is currently a post-doctor researcher in Computer Systems Research Center. His current research interests are high-level synthesis, logic synthesis, and physical design of VLSI circuits, parallel/distributed computing, and network performance analysis. 

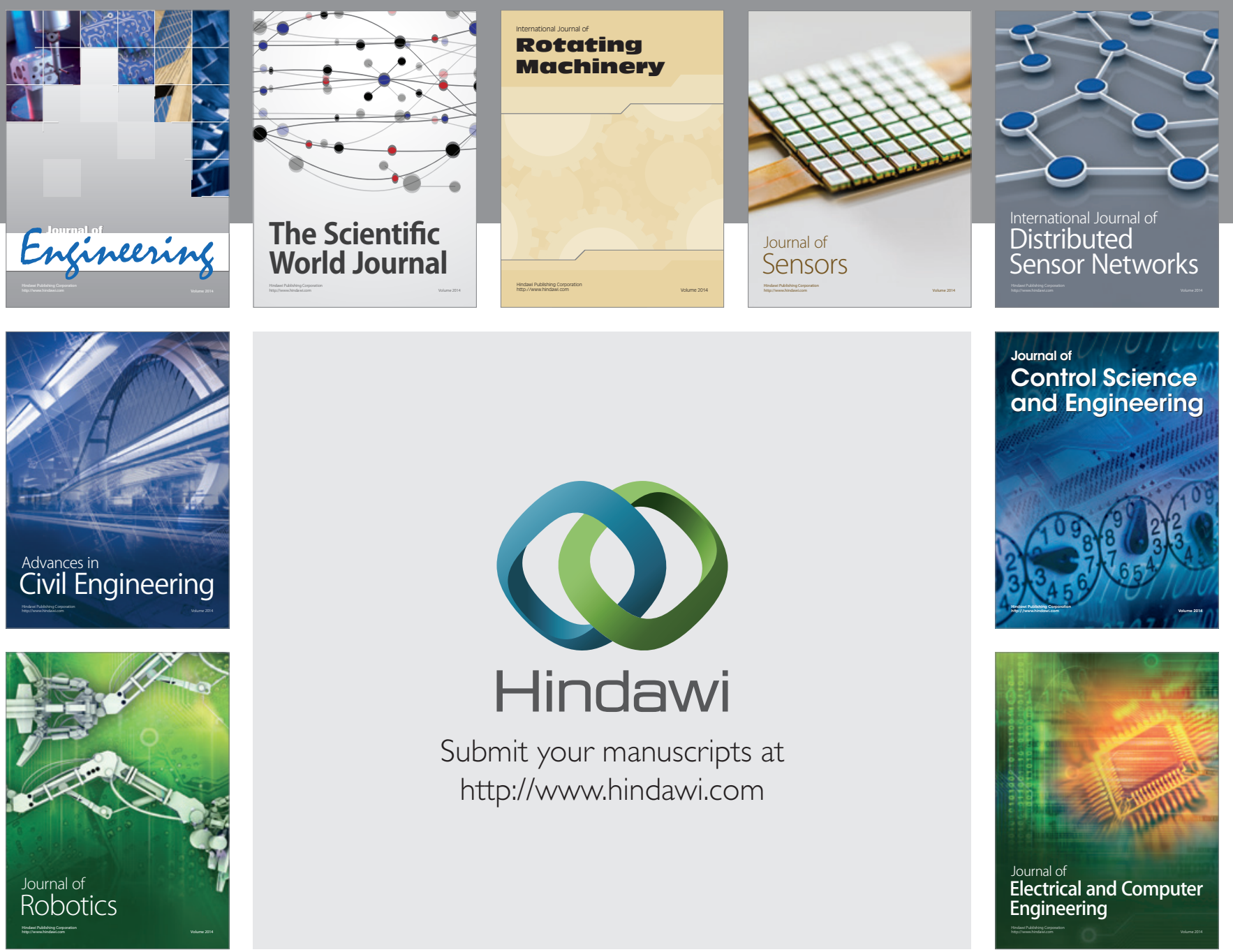

Submit your manuscripts at

http://www.hindawi.com
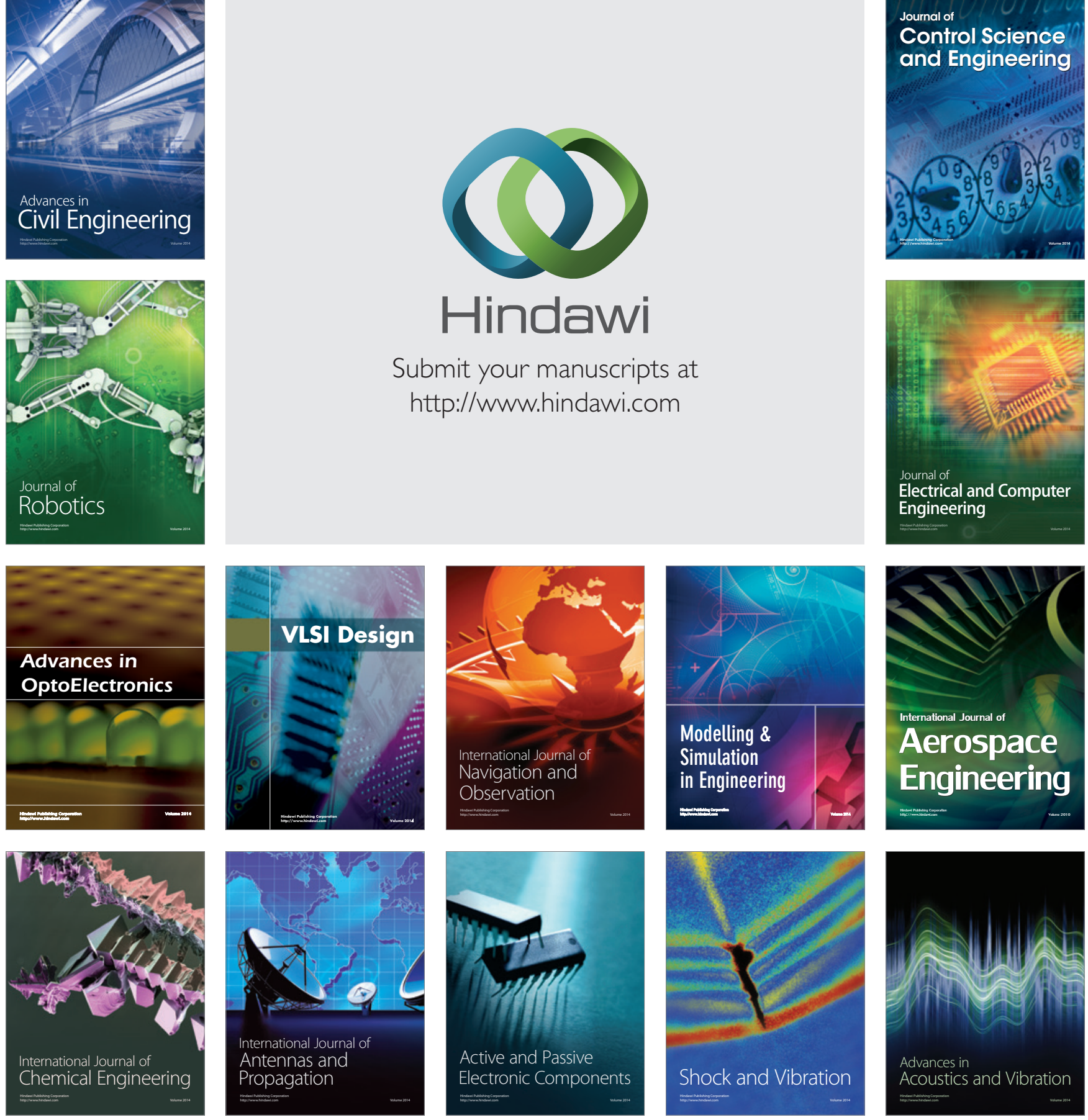